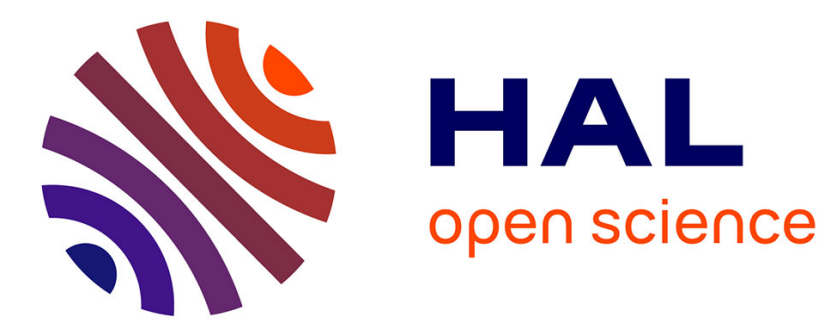

\title{
An algebraic background for hierarchies of PDE in dimension $(2 \mid 1)$
}

Claude Roger

\section{To cite this version:}

Claude Roger. An algebraic background for hierarchies of PDE in dimension (2|1). Pr Odjiewicz. Geometric Methods in Physics, Jun 2016, Bialowieza, Poland. Geometric Methods in Physics (XXXV), Birkhäuser, pp.95-102, 2018, Trends in Mathematics. hal-01406013

\section{HAL Id: hal-01406013 https://hal.science/hal-01406013}

Submitted on 30 Nov 2016

HAL is a multi-disciplinary open access archive for the deposit and dissemination of scientific research documents, whether they are published or not. The documents may come from teaching and research institutions in France or abroad, or from public or private research centers.
L'archive ouverte pluridisciplinaire HAL, est destinée au dépôt et à la diffusion de documents scientifiques de niveau recherche, publiés ou non, émanant des établissements d'enseignement et de recherche français ou étrangers, des laboratoires publics ou privés. 


\title{
An algebraic background for hierarchies of PDE in dimension
}

\section{Claude Roger ${ }^{a}$}

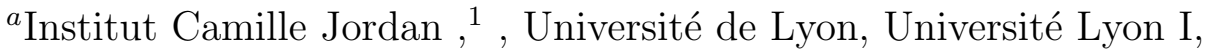
43 boulevard du 11 novembre 1918, F-69622 Villeurbanne Cedex, France
\end{abstract}

Keywords: Schrödinger operators, Superalgebras, Supersymmetry, Hierarchies of PDE.

Mathematics Subject Classification (2000): 17 B65, 34 L30, 35 Q53, 81 T60.

\section{Abstract}

In $d=2$ with variables $(x, t)$, the superalgebraic trick of adding a supplementary odd variable allows the construction of a "square root of time", an operator $D$ satisfying $D^{2}=\partial / \partial t$ in superspace of dimension $(2 \mid 1)$. We already used that trick to obtain a Miura transform in dimension(2|1) for non stationary Schrödinger type operators[5]. We shall discuss here the construction of an algebra of pseudodifferential symbols in dimension $(2 \mid 1)$; that algebra generalizes the one for $d=1$, used in construction of hierarchies from isospectral deformations of stationary Schrödinger type operators.

\section{Reminders on the classic $d=1$ case}

This is the famous Korteweg-De Vries equation and hierarchy, together with its very rich related analytical, geometrical and algebraic structures; they are described in an extensive literature, for example the treatise of Dickey[2], which we shall refer to. It begins with stationary Schrödinger type operators in $d=1$ with variable $x$, like $L=\partial^{2}+u(x)$, then one considers its isospectral deformations, by conjugacy in the space of differential operators or pseudo differential symbols, as follows: $L \rightarrow L_{t}=$ $U(t) L U(t)^{-1}$. The latter formula gives infinitesimally a Lax type equation $\dot{L}=[L, M]$, where as usual, the dot stands for time derivative, so $\dot{L}=\dot{u}$. For suitable values of operator $M$, denoted $M=M_{p}$ for integer $p$, one gets a hierarchy of equations $\dot{L}=\left[L, M_{p}\right]$. If moreover, the operators $M_{p}$ are mutually commuting $\left[M_{p}, M_{q}\right]=0$, one deduces an infinite family of conserved quantities for those equations, then said to be completely integrable system with an infinite number of degrees of freedom.

\footnotetext{
${ }^{1}$ Laboratoire associé au CNRS UMR 5208
} 
Those equations are hamiltonian for the Poisson structure of the dual of Virasoro algebra (cf.[3]) of vector fields $u(x) \partial$, with the well known bracket $[u(x) \partial, v(x) \partial]=$ $\left(u(x) v^{\prime}(x)-v(x) u^{\prime}(x)\right) \partial$, and central term; from that Lie algebra one constructs the associative algebra $O D$ of differential operators of the form $u_{0}+u_{1} \partial+\ldots .+u_{n} \partial^{n}$, and finally, by formally inverting $\partial$ into $\partial^{-1}$, one gets the division algebra of pseudo differential symbols $\Psi D$. A symbol $D \in \Psi D$ has the form

$$
D=\sum_{n=-\infty}^{n=N} a_{n} \partial^{n} .
$$

Associative algebra $\Psi D$ encodes all computations necessary for construction of operators $M_{p}$, and also the right Poisson structures for those hamiltonian equations, the dual of Virasoro algebra and its higher order generalizations known as $\mathcal{W}$-algebras. Let's recall that the key formula for algebraic calculations in $\Psi D$ is the following:

$$
\partial^{-1} a=\sum_{n=0}^{\infty}(-1)^{n} \partial^{n}(a) \partial^{-1-n} .
$$

That algebra allows computation of square root of $L=\partial^{2}+u(x)$, and further its successive half-integer powers, giving hierarchies and conserved quantities. As well,Miura's transform factorizes $\partial^{2}+u(x)=(\partial+v(x)) \circ(\partial-v(x)$ and turns out to be a rather useful tool.

\section{Non stationary Schrödinger operators}

Now, we are in the $d=2$ case with variables $(x, t)$, let's set for short $\frac{\partial}{\partial x}=\partial \frac{\partial}{\partial t}=\partial_{t}$; we shall try to generalize the constructions described in part 1 above to non stationary operators of Schrödinger type with potential: $\mathfrak{S} \mathfrak{c h} \mathfrak{r}=\partial^{2}-\partial_{t}+u(x, t)$.

\section{Remarks:}

1/One may think of the space parametrized by $(x, t)$ as spacetime with space dimension 1 , the variable $t$ being the physical time, but it is not necessarily the case.

$2 /$ Those operators are, strictly speaking, heat operators, but Wick rotation $t \rightarrow i t$ transforms them into the actual Schrödinger operator.

3/ Hierarchies of PDE in dimension 2 and more have been on the agenda since some time, with various point of view, cf. for example [8].

In the present paper, we shall use a supersymmetric trick: we enlarge the space with an odd dimension and work in the superspace of dimension (2|1), parametrized with even variables $(x, t)$ and one odd variable $\theta$. The even variables can be polynomial, 
analytic or differentiable, we can have as well $x \in \mathbb{R}$ or $x \in S^{1}$, and the same for $t$. We shall not make things more precise for the moment, and all necessary technical preliminaries on superalgebra will be developed in the next part.

We shall now consider the odd differential operator $D_{\theta}=\theta \partial_{t}+\partial_{\theta}$, which satisfies $D_{\theta}^{2}=\partial_{t}$; so $D_{\theta}$ represents the promised "square root of time", and we can write non stationary Schrödinger operator as a difference of two squares $\mathfrak{S} \mathfrak{c h} \mathfrak{r}=\partial^{2}-D_{\theta}^{2}+u(x, t)$.

\section{Some techniques of graded algebra}

We shall recall in this part the most basic definitions and formulas for graded algebra, a detailed introduction can be found in[1]. A graded (or super-) algebra is an associative algebra which admits a graduation, following $\mathcal{A}=\oplus_{p \in \mathbb{Z}} A^{p}$; an element $a \in A^{p}$ is said to be of degree $p$, denoted by $|a|=p$. The associative multiplication is graded, i.e.

$$
|a b|=|a|+|b|
$$

and supercommutative (or graded commutative) which means:

$$
a b=(-1)^{|a||b|} b a .
$$

So, $a$ and $b$ anticommute iff they are both of odd degree, otherwise they commute.

The typical examples are the free algebras on even generators $x_{i}$ and odd generators $\theta_{\alpha}$, written as

$$
\mathcal{A}=k\left[x_{i}\right] \otimes \Lambda\left(\theta_{\alpha}\right)
$$

where as usual the symbol $\Lambda$ denotes exterior algebra, and $k\left[x_{i}\right]$ the polynomial algebra in indeterminates $x_{i}$, with coefficients in a field of vanishing characteristic $k$; in most examples $k=\mathbb{C}$. One can also give a more global description of those superalgebras : consider two finite dimensional vector spaces $E_{0}$ and $E_{1}$ and assume that elements of $E_{0}$ (resp. $\left.E_{1}\right)$ are even (resp. odd), then the free supercommutative algebra on $E_{0} \oplus E_{1}$ will be

$$
\mathcal{A}=S^{*}\left(E_{0}\right) \oplus \Lambda^{*}\left(E_{1}\right) .
$$

In most cases only modulo 2 degree is taken in account, being the only relevant part for sign formulas.

A map $f$ between graded spaces has a degree $|f|$ naturally defined by $|f(a)|=$ $|f|+|a|$, and we shall consider derivations of graded algebras. A map $\delta: \mathcal{A} \rightarrow \mathcal{A}$ is a derivation if for any $a, b \in \mathcal{A}$, one has:

$$
\delta(a b)=\delta(a) b+(-1)^{|a||\delta|} a \delta(b) .
$$


So one has odd and even derivations. Now we can consider the graded commutator of derivations following the formula:

$$
\left[\delta_{1}, \delta_{2}\right]=\delta_{1} \circ \delta_{2}-(-1)^{\left|\delta_{1}\right|\left|\delta_{2}\right|} \delta_{2} \circ \delta_{1}
$$

This bracket defines a Lie superalgebra structure on the space of derivations (see[1] for precise formalism about Lie superalgebras); the geometric interpretation of derivations as tangent vector fields allows to consider the space of derivations $\operatorname{Der}(\mathcal{A})$ as the Lie superalgebra of tangent vector fields on the underlying super manifold whose space of functions is $\mathcal{A}[6]$. Finally, we shall make use for any graded algebra, of the following involution: $a \rightarrow \bar{a}=(-1)^{|a|} a$.

\section{The division algebra of pseudodifferential symbols in $d=$ $(2 \mid 1)$}

Our differential and pseudo differential operators will act on the superalgebra $\mathcal{A}$ of functions on superspace of dimension $(2 \mid 1)$. We shall use as generators the following two operators: $\mathcal{D}=\partial+i D_{\theta}$ and $\overline{\mathcal{D}}=\partial-i D_{\theta}$, where $i$ is present mainly for technical reasons. Computations are a bit delicate, since

$$
\mathcal{D}(a b)=\mathcal{D}(a) b+a \mathcal{D}(b)
$$

if $a$ is even, but

$$
\mathcal{D}(a b)=\mathcal{D}(a) b+a \overline{\mathcal{D}}(b)
$$

if $a$ is odd. In terms of composition of operators, an element $a \in \mathcal{A}$ being viewed as an operator of order zero, one has

$$
\mathcal{D} \circ a=a \circ \mathcal{D}+\mathcal{D}(a)
$$

if $|a|=0(\bmod .2)$, and

$$
\mathcal{D} \circ a=a \circ \overline{\mathcal{D}}+\mathcal{D}(a)
$$

if $|a|=1$ (mod.2). This is why we cannot consider operators in powers of $\mathcal{D}$ only, we must add the conjugate $\overline{\mathcal{D}}$ in order to get a closed algebra.

Those elements generate the associative graded algebra of differential operators on $\mathcal{A}$, denoted as $O D(\mathcal{D}, \overline{\mathcal{D}})$. Its generic element has the following form:

$$
\sum_{k \geq 0, l \geq 0}^{\text {finite }} a_{k, l} \mathcal{D}^{k} \overline{\mathcal{D}}^{l}
$$


We can change the generators from $(\mathcal{D}, \overline{\mathcal{D}})$ to $\left(\partial, D_{\theta}\right)$, and so $O D(\mathcal{D}, \overline{\mathcal{D}})=O D\left(\partial, D_{\theta}\right)$. Straightforward computations give easily:

$$
\begin{aligned}
& \mathcal{D}^{2}=\partial^{2}-\partial_{t}+2 i \partial D_{\theta} \\
& \overline{\mathcal{D}}^{2}=\partial^{2}-\partial_{t}-2 i \partial D_{\theta}
\end{aligned}
$$

so

$$
\frac{\mathcal{D}^{2}+\overline{\mathcal{D}}^{2}}{2}=\partial^{2}-\partial_{t}=\mathfrak{S c h h}_{0}
$$

the non stationary Schrödinger operator with zero potential. This is why algebra $O D(\mathcal{D}, \overline{\mathcal{D}})$ is relevant for our problem! Moreover, one has $\mathcal{D} \overline{\mathcal{D}}=\overline{\mathcal{D}} \mathcal{D}=\partial^{2}+\partial_{t}$, and omitting the $i$ coefficient, we could have obtained $\partial^{2}-\partial_{t}$, like in[6], where that formula was used for generalization of Miura transform.

We are now ready to construct the algebra of pseudo differential symbols $\Psi D(\mathcal{D}, \overline{\mathcal{D}})$ by localization of $O D(\mathcal{D}, \overline{\mathcal{D}})$; let's invert formally $\mathcal{D}$ and $\overline{\mathcal{D}}$ in $\mathcal{D}^{-1}$ and $\overline{\mathcal{D}}^{-1}$ respectively, so a generic pseudodifferential symbol will have the form:

$$
\sum_{k=-\infty, l=-\infty}^{\text {finite }} a_{k, l} \mathcal{D}^{k} \overline{\mathcal{D}}^{l} .
$$

As in the $d=1$ case, the difficulty is to establish a coherent formula for the composition $\mathcal{D}^{-1} \circ a$; we shall prove by recurrence on the degree that :

$$
\mathcal{D}^{-1} \circ a=\sum_{i, j=0 ; i+j>0}^{+\infty} a_{i, j} \mathcal{D}^{-i} \overline{\mathcal{D}}^{-j} .
$$

(Here we omitted symbol $\circ$ when obvious). As in the $d=1$ case the formulas will be obtained from $\mathcal{D} \circ \mathcal{D}^{-1} \circ a=a$; we shall use decomposition of any element $a \in \mathcal{A}$ according parity, $a=a^{0}+a^{1}$. We shall proceed by recurrence, from:

$$
a=\mathcal{D}\left(\sum_{i, j=0 ; i+j>0}^{+\infty} a_{i, j} \mathcal{D}^{-i} \overline{\mathcal{D}}^{-j}\right),
$$

so:

$$
\mathcal{D}\left(a_{i, j} \mathcal{D}^{-i} \overline{\mathcal{D}}^{-j}\right)=\mathcal{D}\left(a_{i, j}\right) \mathcal{D}^{-i} \overline{\mathcal{D}}^{-j}+a_{i, j}^{0} \mathcal{D}^{-i+1} \overline{\mathcal{D}}^{-j}+a_{i, j}^{1} \mathcal{D}^{-i} \overline{\mathcal{D}}^{-j+1}
$$

One then readily deduce from above the recurrence formula

$$
\mathcal{D}\left(a_{i, j}\right)=-a_{i+1, j}^{0}-a_{i, j+1}^{1}
$$

The recurrence is easily introduced: if $a=a^{0}+a^{1}$, then $a_{1,0}=a_{1,0}^{0}=a^{0}$ and $a_{0,1}=$ $a_{0,1}^{1}=a^{1}$; so one gets a unique solution to the equation above but the general formulas 
for $a_{i, j}$ are not obvious to be made explicit; for example one has $a_{i+1,0}=(-1)^{i} \partial^{i} a^{0}$, and also $a_{0, j+1}=(-1)^{j} \partial^{j} a^{1}$; we shall give also some samples in low degree: $a_{1,1}=-i D_{\theta} a$, $a_{1,2}=i \partial D_{\theta} a-\partial_{t} a^{1}$ and $a_{2,1}=i \partial D_{\theta} a-\partial_{t} a^{0}$. Computations are exactly parallel when one computes $\overline{\mathcal{D}}^{-1} \circ a$.

Remark: In [7] we considered algebras of differential operators and pseudodifferential symbols in dimension(1|1) with variables $(x, \theta)$, variable $t$ being a loop space coordinate; in [9] the authors give a detailed study of the algebra of differential operators in dimension (1|1), they award its paternity to Manin and Radul [4] in their work about SuSy (supersymmetric) extension of KP hierarchy. From $D_{\theta}^{2}=\partial_{t}$, one can consider $O D(\mathcal{D}, \overline{\mathcal{D}})=O D\left(\partial, D_{\theta}\right)$ as a quadratic extension of $O D\left(\partial, \partial_{1}\right)$ which is simply the algebra of differential operators in $d=2$; but those algebras are neither commutative nor anticommutative. Let's stress also the fact that $O D(\mathcal{D}, \overline{\mathcal{D}})=O D\left(\partial, D_{\theta}\right)$ doesn't imply that $\Psi D(\mathcal{D}, \overline{\mathcal{D}})=\Psi D\left(\partial, D_{\theta}\right)$, since different choice of generators to be inverted change the global algebraic structure.

\section{Schrödinger type operators in dimension $(2 \mid 1)$}

We shall consider operators of the type $\mathcal{D}^{2}+\mathcal{U}$, where potential $\mathcal{U}=u+i \theta \phi$ has an odd and even part. Moreover, $\mathcal{D}^{2}=\partial^{2}-\partial_{t}+2 i \partial D_{\theta}=\mathfrak{S} \mathfrak{c h} \mathfrak{r}_{0}+2 i \partial D_{\theta}$. We get an unwanted supplementary term, it can be considered as the "price to pay" for generalization to $d=2$, like in the case of Miura transform [5].

The wave functions must be looked for among super functions as $F=f+i \theta \alpha$ and spectral values have odd and even part too: $\Lambda=\lambda+i \theta \xi$. One has

$$
\mathcal{D}^{2}(F)=\mathfrak{S} \mathfrak{c h} \mathfrak{r}(f)+i \theta \mathfrak{S} \mathfrak{c h} \mathfrak{r}(\alpha)+2 i \partial D_{\theta}(F)
$$

then one readily computes $D_{\theta}(F)=\theta \partial_{t} f+i \alpha$. Then:

$$
\left(\mathcal{D}^{2}+\mathcal{U}\right)(F)=\mathfrak{S} \mathfrak{c h} \mathfrak{r}(f)+u f-2 \partial \alpha+i \theta\left(\mathfrak{S} \mathfrak{c h} \mathfrak{r}(\alpha)+u \alpha+2 \partial \partial_{t} f+\phi f\right)
$$

Finally, equation

$$
\left(\mathcal{D}^{2}+\mathcal{U}\right)(F)=\Lambda F
$$

induces the following system:

$$
\begin{gathered}
\mathfrak{S} \mathfrak{c h} \mathfrak{r}(f)+u f-2 \partial \alpha=\lambda f \\
\left.\mathfrak{S} \mathfrak{c h} \mathfrak{r}(\alpha)+u \alpha+2 \partial \partial_{t} f+\phi f\right)=\lambda \alpha+\xi f .
\end{gathered}
$$

In the particular case when the wave function $F$ is purely even, so $F=f$, one obtains a coupled system in $f$ :

$$
\mathfrak{S} \mathfrak{c h} \mathfrak{r}(f)+u f=\lambda f
$$




$$
2 \partial \partial_{t} f+\phi f=\xi f .
$$

The second equation is of Klein-Gordon type, so the system couples hyperbolic and parabolic system.

\section{$6 \quad \mathrm{KdV}$ like equations in $d=2$}

We shall obtain evolution equations w.r.t. a real parameter $s$ on a pair of functions $(u, \phi)$ in variables $(x, t, s)$ appearing as components of a super function $\mathcal{U}=u+i \theta \phi$ as above. We now develop a formalism parallel to the classical $d=1$ case[2], and thus consider the equation:

$$
4 \mathcal{U}_{s}=\mathcal{D}^{3}+6 \mathcal{U} \mathcal{D}(\mathcal{U}),
$$

One deduces from it the following system of evolution PDE:

$$
\begin{gathered}
u_{s}=\partial^{3} u-3 \partial \partial_{t} u+6 u \partial u-6 u \phi-3 \partial^{2} \phi+\partial_{t} \phi \\
\phi_{s}=\partial^{3} \phi-3 \partial \partial_{t} \phi+3 \partial^{2} \partial_{t} u-\partial_{t}^{2} u+6 \phi \partial u+6 u \partial \phi-6 \phi^{2}+6 u \partial_{t} u
\end{gathered}
$$

Let's now consider the case when the potential $\mathcal{U}$ is purely even, when $\phi=0$. The second equation yields :

$$
3 \partial^{2} \partial_{t} u-\partial_{t}^{2} u+6 u \partial_{t} u=0
$$

SO:

$$
\partial_{t}\left(3 \partial^{2} u-\partial_{t} u+3 u^{2}\right)=0 .
$$

One deduces immediately:

$$
3 \partial^{2} u-\partial_{t} u+3 u^{2}=f(x)(*)
$$

where $f$ is an arbitrary function independent of $t$. The first equation gives :

$$
u_{s}=\partial^{3} u-3 \partial \partial_{t} u+6 u \partial u
$$

. Now by deriviation of equation $(*)$, one obtains

$$
3 \partial^{3} u-\partial \partial_{t} u+6 u \partial u=\partial f(x)
$$

and we can cancel the non linear term, getting the linear dispersive equation with sources:

$$
u_{s}=-2 \partial^{3} u-2 \partial \partial_{t} u+\partial f(x)(* *)
$$


Another option is to cancel the term with $\partial_{t}$ between $\left(^{*}\right)$ ans the first equation, and one gets an equation in $(x, s)$ without $t$ as follows:

$$
u_{s}=-8 \partial^{3} u-12 u \partial u+3 \partial f(x) ;
$$

after change of scale $s \rightarrow-s$ and $x \rightarrow 2 x$, one obtains finally:

$$
u_{s}=\partial^{3} u+6 u \partial u-\frac{3}{2} \partial f(x),
$$

which is exactly Korteweg De Vries equation with source.

Now, if $\mathcal{U}$ is purely odd, i.e. $u=0$, the above system reduces to:

$$
\begin{gathered}
3 \partial^{2} \phi=\partial_{t} \phi \\
\phi_{s}=\partial^{3} \phi-3 \partial \partial_{t} \phi-6 \phi^{2} .
\end{gathered}
$$

Finally, cancellation of terms in $\partial_{t}$ will give the following equation in $(x, s)$ :

$$
\phi_{s}+8 \partial^{3} \phi+6 \phi^{2}=0 .
$$

Any solution of that equation in $(x, s)$ can be prolongated to a general solution in $(x, s, t)$, simply by using a heat kernel.

\section{References}

[1] Pierre Deligne and John W. Morgan. Notes on supersymmetry (following Joseph Bernstein). In Quantum fields and strings: a course for mathematicians, Vol. 1, 2 (Princeton, NJ, 1996/1997), pages 41-97. Amer. Math. Soc., Providence, RI, 1999.

[2] L. A. Dickey. Soliton equations and Hamiltonian systems, volume 26 of Advanced Series in Mathematical Physics. World Scientific Publishing Co., Inc., River Edge, NJ, second edition, 2003.

[3] Laurent Guieu and Claude Roger. L'algèbre et le groupe de Virasoro. Les Publications CRM, Montreal, QC, 2007. Aspects géométriques et algébriques, généralisations. [Geometric and algebraic aspects, generalizations], With an appendix by Vlad Sergiescu.

[4] Yu. I. Manin and A. O. Radul. A supersymmetric extension of the KadomtsevPetviashvili hierarchy. Comm. Math. Phys., 98(1):65-77, 1985.

[5] Claude Roger. A matrix Miura transform. J. Geom. Phys., 74:87-90, 2013. 
[6] Claude Roger. Algebras generated by odd derivations. J. Geom. Symmetry Phys., 40:53-59, 2015.

[7] Claude Roger and Jérémie Unterberger. A Hamiltonian action of the SchrödingerVirasoro algebra on a space of periodic time-dependent Schrödinger operators in (1+1)-dimensions. J. Nonlinear Math. Phys., 17(3):257-279, 2010.

[8] Artur Sergyeyev and Błażej M. Szablikowski. Central extensions of cotangent universal hierarchy: $(2+1)$-dimensional bi-Hamiltonian systems. Phys. Lett. A, 372(47):7016-7023, 2008.

[9] Theodore Voronov Simon Li, Ekaterina Shemyakova. Differential operators on the superline, berezinians, and darboux transformations. arXiv, 1605.07286, 2016. 